\title{
Use of simultaneous fluoroscopic and echocardiographic guidance during transarterial coil placement for embolization of patent ductus arteriosus in dogs
}

\author{
Viktor Szatmári, DVM, PhD, and Arnold A. Stokhof, DVM, PhD
}

\begin{abstract}
Objective-To evaluate the use of simultaneous fluoroscopic and transthoracic echocardiographic guidance during transarterial coil placement for embolization of patent ductus arteriosus (PDA) in dogs.
\end{abstract}

Design-Descriptive report.

Animals-3 dogs with PDA.

Procedure-Each dog was anesthetized, and a femoral artery was exposed for vascular access. By use of an introducer, a catheter was placed in the thoracic portion of the descending aorta with fluoroscopic guidance, and subsequently, a bolus of iodinated radiographic contrast material was injected to outline the ductus. Under fluoroscopic guidance, 1 coil was positioned in the ductus, but not released. Transthoracic echocardiography was used to ensure that 1 loop of the coil was located in the pulmonary artery. When $>1$ loop or no loops were detected in the pulmonary artery, the coil was retrieved and repositioned; when 1 loop of the coil was detected in the pulmonary artery, the coil was detached. After catheter removal, the femoral artery was ligated and the wound was closed.

Results_-In all 3 dogs, successful embolization of the PDA was achieved. Echocardiography prevented unintentional pulmonary artery embolization in 1 dog and suboptimal coil placement in the other 2 dogs.

Conclusions and Clinical Relevance-In addition to fluoroscopic control, transthoracic echocardiography appears to aid the appropriate positioning of a transarterial coil for treatment of PDA in dogs. Although transesophageal echocardiography would likely provide better images of the ductus, transthoracic echocardiography is a much cheaper, less specialized, and more widely available alternative. (J Am Vet Med Assoc 2006;228:881-884)

$\mathbf{P}$ atent ductus arteriosus represents the most common congenital cardiac anomaly in dogs. ${ }^{1}$ In instances of left-to-right shunting (ie, blood flow from the descending aorta to the pulmonary artery), closure of the PDA stops the detrimental effects of left ventricular volume overload and offers an excellent prognosis provided that left-sided heart failure or atrial fibrillation has not developed yet. ${ }^{2}$

The traditional treatment is surgical ligation of the PDA via thoracotomy. ${ }^{2}$ Coil embolization of the PDA is a minimally invasive procedure that has gained increasing

\footnotetext{
From the Department of Clinical Sciences of Companion Animals, Faculty of Veterinary Medicine, Utrecht University, Yalelaan 8, 3584 CM, Utrecht, The Netherlands.

Address correspondence to Dr. Szatmári.
}

popularity during the past decade.$^{3-6}$ Compared with thoracic surgery, coil embolization is not associated with possibly fatal intraoperative hemorrhage (as a result of PDA laceration), formation of pleural adhesions, or complications in wound healing; moreover, signs of pain after the procedure are likely to be reduced. The disadvantages of coil embolization are exposure of medical personnel and patient to radiation as well as the potential for increased duration of anesthesia, although the time required to complete the procedure is highly operator dependent. Possible complications of coil placement include intravascular hemolysis, ${ }^{7}$ dislocation of the coil into the pulmonary artery ${ }^{4,8}$ or the aortic tree, ${ }^{9}$ and severe hemorrhage from the arterial puncture site if the Seldinger technique is used..$^{10}$ A further complicating factor of coil placement is the need for various devices and methods according to the size and shape of the PDA. ${ }^{11}$ This requires the operator to be familiar with a variety of embolization devices.

Dislocation of the coil into the pulmonary artery tree occurs with a frequency of 3\% to $46 \%$. $^{4,8}$ Although the presence of a coil in the pulmonary artery vasculature does not seem to have clinical consequences, ${ }^{8}$ another effort has to be made to close the shunt after an unsuccessful coil placement; this potentially involves prolonged anesthesia of the dog, prolonged radiation exposure of personnel and patient, and additional costs for the owner, and the dog may also have to undergo thoracic surgery. ${ }^{4,8}$

Selection of a coil of appropriate size is made on the basis of angiographic findings ${ }^{4}$ or the combined results of transthoracic ultrasonography before and angiography

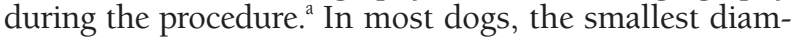
eter of the ductus and the diameter of the ampulla can be determined via examination of standard transthoracic echocardiographic images-the long-axis view in left cranial parasternal position and the short-axis view in right parasternal location. ${ }^{12}$ According to the manufacturer's recommendation, the coil's diameter should be at least twice the size of the smallest diameter of the ductus, and 1 loop should be positioned in the pulmonary artery.

We hypothesized that unintentional pulmonary embolizations result from 1 of 2 possible misjudgments during coil placement. First, if the size of the PDA is larger than estimated from the lateral angiographic images, the coil will not stay in place despite correct positioning because the high aortic pressure pushes it into the pulmonary artery. The second possibility is that although an appropriately sized coil has

\section{PDA Patent ductus arteriosus}

MPA Main pulmonary artery 
been selected, the tip of the delivery catheter is placed in the pulmonary artery and not in the ductus, which results in release of the coil in the lumen of the pulmonary artery. Because the pulmonary artery can easily be visualized via transthoracic echocardiography, ${ }^{12}$ we hypothesized that ultrasonographic control of the fluoroscopically positioned, but not yet released, coil could eliminate the latter possible cause of pulmonary artery embolization. The purpose of the study reported here was to evaluate the use of simultaneous fluoroscopic and echocardiographic guidance during transarterial coil placement for embolization of PDAs in dogs.

\section{Procedure}

The procedure was performed in anesthetized dogs. Dogs were premedicated with methadone $(0.5 \mathrm{mg} / \mathrm{kg}$ $[0.23 \mathrm{mg} / \mathrm{lb}], \mathrm{IV})$ and midazolam $(0.3 \mathrm{mg} / \mathrm{kg}[0.14$ $\mathrm{mg} / \mathrm{lb}], \mathrm{IV}$ ). Anesthesia was induced with propofol (2 to $3 \mathrm{mg} / \mathrm{kg}$ [0.91 to $1.36 \mathrm{mg} / \mathrm{lb}$ ], IV, to effect) and after intratracheal intubation was maintained with $1 \%$ isoflurane vaporized in 100\% oxygen. Intraoperative analgesia was provided via administration of sufentanil $(1 \mu \mathrm{g} / \mathrm{kg} / \mathrm{h}$ $[0.45 \mu \mathrm{g} / \mathrm{lb} / \mathrm{h}], \mathrm{IV})$. Atropine $(0.02 \mathrm{mg} / \mathrm{kg}$ [0.01 mg/lb], IM or IV) was administered when the heart rate was $<80$ beats/min. Continuous ECG, pulse oximetry, capnography, and direct arterial blood pressure measurement were used to monitor dogs during anesthesia.

Each dog was placed in right lateral recumbency on the angiography table. The skin on the medial aspect of the right thigh was aseptically prepared (ie, shaved and disinfected) and isolated by use of surgical drapes. The skin on the cranial part of the left thoracic wall was similarly prepared, and a hole in the isolating drape was made to allow direct contact between the ultrasound probe and the skin. On the medial side of the right femur, the femoral artery was exposed via a surgical cut down procedure, and its distal end was ligated. A 5-F introducer with a hemostatic valve was inserted in the proximal end of the femoral artery and secured to the skin with 2 ligatures. A 5-F pigtail catheter was introduced through the introducer into the descending aorta; the tip of the catheter was advanced to the level of the tracheal bifurcation.

To delineate the ductus, angiography was performed by rapid hand injection of water-soluble iodinated contrast agent ${ }^{\mathrm{b}}(1 \mathrm{~mL} / \mathrm{kg})$. The angiogram was recorded on videotape, and the smallest and largest widths as well as the length of the PDA were estimated on the recorded images with the aid of a calibrated radiopaque ruler placed under the dog. After removal of the pigtail catheter, a 5-F multipurpose end-hole catheter was advanced through the introducer into the aorta and through the ductus into the MPA. The pressures were measured through the catheter with a pressure transducer and recorded for all 3 vessels (ie, MPA, PDA, and aorta). Thereafter, the tip of the catheter was guided into the ductus by monitoring the angiogram and simultaneous pressure recordings.

A detachable coil ${ }^{c}$ was then advanced and pushed out of the catheter after having withdrawn the straightening mandril. When the position of the coil was thought to be correct on the basis of the fluoroscopic images, the MPA was identified via the left cra- nial thoracic wall by use of a 4- to 7-MHz electronic sector ultrasound probe. ${ }^{d}$ Sterile infusion $(0.9 \% \mathrm{NaCl})$ solution was used to make contact between the transducer and the skin. First, the aorta was viewed with the standard long-axis view in the left cranial parasternal location. ${ }^{12}$ From this position, the transducer was angled dorsally to obtain images of the MPA with the standard long-axis view in the left cranial parasternal location. ${ }^{12}$ The transducer and the keyboard of the ultrasound machine were covered with sterile material, ${ }^{e}$ enabling the operator to perform the echocardiographic examination without contaminating the surgical field. The coil was positioned so that 1 loop would be visible in the pulmonary artery via transthoracic echocardiography. When this stage had been reached, the anesthesiologist was asked to auscultate the heart of the dog to confirm the disappearance of the continuous murmur. At this time, the coil was detached under fluoroscopic control. After removal of the delivery catheter, the pigtail catheter was introduced again, and 10 minutes after coil placement, the angiographic procedure was repeated. Then, the proximal end of the femoral artery was ligated after removal of the catheter and the introducer. The subcutis and skin were closed with sutures in an interrupted pattern. Each dog was sent home within 24 hours after the intervention, and a recheck evaluation was scheduled 6 weeks later.

\section{Results}

The procedure described in this report was applied in 3 client-owned dogs. Informed consent was obtained from the owners prior to the procedure. All 3 dogs had no clinical signs of disease, and each was referred because a cardiac murmur was detected during routine physical examination. Patent ductus arteriosus was suspected on the basis of physical findings (waterhammer-quality femoral pulse and continuous murmur with a precordial thrill that had a point of maximum intensity in the region of the pulmonary artery valve) and was confirmed via B-mode and color flow Doppler echocardiography (detection of a dilatated left atrium and ventricle and dilatated MPA with a reversed jet originating from the pulmonary artery bifurcation toward the pulmonic valves, and direct identification of the PDA). No concomitant cardiac anomalies were detected in any of the dogs.

One coil with a diameter of $5 \mathrm{~mm}$ and 3 loops was positioned in the PDA of a 3-month-old female West Highland White Terrier (weight, $2.2 \mathrm{~kg}$ [4.84 lb]), 1 coil with a diameter of $8 \mathrm{~mm}$ and 5 loops was positioned in the PDA of a 6-month-old male Cavalier King Charles Spaniel (weight, $4.1 \mathrm{~kg}$ [9.02 lb]), and 1 coil with a diameter of $8 \mathrm{~mm}$ and 5 loops was positioned in the PDA of a 3-month-old female Slovakian hound (weight, $7.5 \mathrm{~kg}[16.5 \mathrm{lb}]$ ). Recovery from anesthesia was unremarkable in all 3 dogs, and no complications were detected during the postoperative period.

In all 3 dogs, the MPA was identified echocardiographically via the left thoracic wall. The contour of the coil appeared hyperechoic on echocardiographic images (Figure 1); the number of loops could clearly be seen in real-time images. 


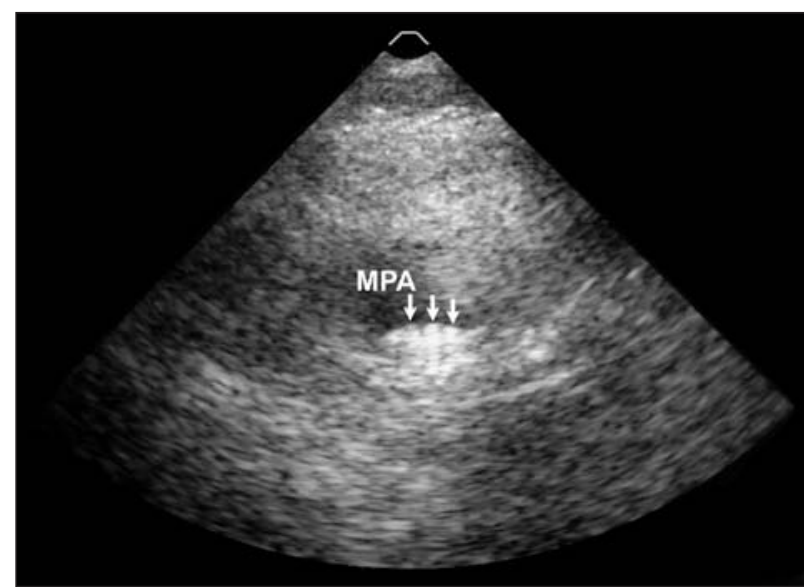

Figure 1-B-mode transthoracic echocardiographic image (standard long-axis view; left cranial parasternal location) obtained via the left cranial aspect of the thoracic wall of a West Highland White Terrier positioned in right lateral recumbency during coil embolization of a PDA. All 3 loops (each of them indicated by an arrow) of the 3-loop coil can be seen as hyperechoic structures in the MPA. This fact necessitated coil repositioning before its release. Although the MPA is unrecognizable on this static image, it extends vertically in this view; the pulmonic valves are located toward the top and the bifurcation toward the bottom of this image. The coil appears in the MPA immediately proximal to the bifurcation.

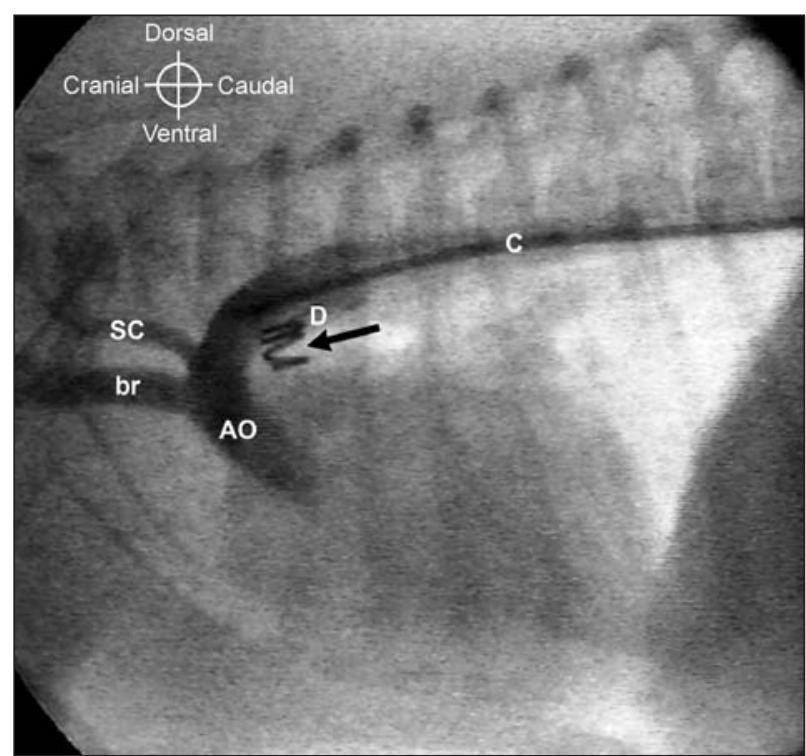

Figure 2-Angiographic image of the PDA (D) of the West Highland White Terrier in Figure 1 after correct placement of the coil. The gap between the most ventral loop and the rest of the coil (arrow) indicates proper placement. The gap is caused by the walls of the ductus and the pulmonary artery. The loop ventral to the arrow is located in the MPA, and the 2 loops dorsal to the arrow are located in the ductus. $\mathrm{C}=$ Catheter in the aorta (AO). $\mathrm{br}=$ Brachiocephalic trunk. SC $=$ Left subclavian artery.

In the West Highland White Terrier, transthoracic echocardiography revealed that all 3 loops of the coil were located in the MPA when the position of the coil was thought to be correct on the basis of the recorded angiographic images and pressure measurements (Figure 1). On the basis of the echocardiographic images, a decision was made to reposition the coil. After the coil was pulled back into the catheter, the catheter tip was pulled back a small distance and the

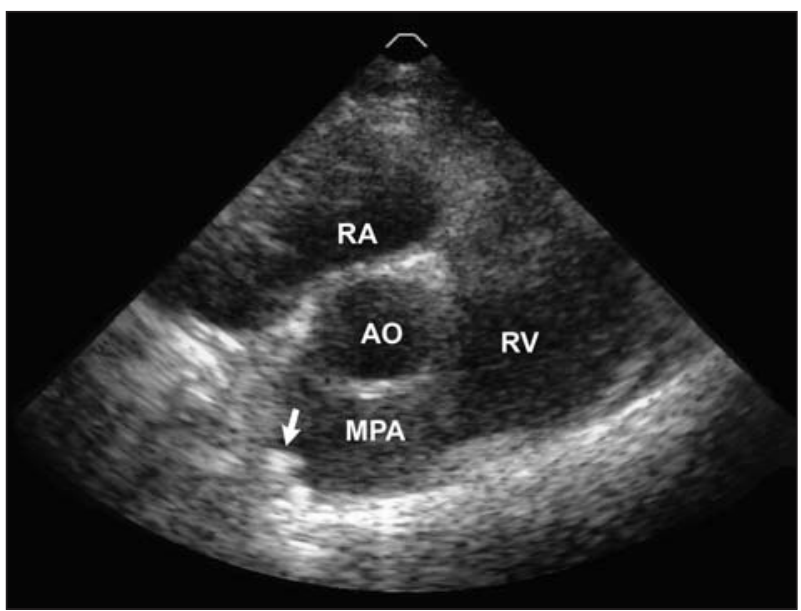

Figure 3-Standard B-mode short-axis right parasternal transthoracic echocardiographic image of the MPA via the right thoracic wall of the dog in Figure 1 obtained 6 weeks after coil embolization of the PDA. The dog was in right lateral recumbency. One loop of the coil (arrow) is detectable in the MPA as a hyperechoic structure at the point of the pulmonic bifurcation. $\mathrm{RA}=$ Right atrium. $\mathrm{RV}=$ Right ventricle. See Figure 2 for remainder of key.

coil was pushed out again. After this, no parts of the coil were seen in the MPA immediately proximal to the bifurcation, suggesting that the entire coil was either in the ductus or in the aorta. After the coil was pulled back into the catheter again and the catheter repositioned (ie, advanced a short distance), 1 loop of the coil appeared in the MPA. At this time, no audible murmur was detected and the coil was detached under fluoroscopic guidance; the coil subsequently stayed in its position. Angiography was performed 10 minutes later and revealed slight residual shunting through the properly placed coil (Figure 2). At a recheck examination 6 weeks later, echocardiography revealed no flow through the ductus. One loop of the coil was easily recognizable in the MPA at the point of the bifurcation (Figure 3).

In the other 2 dogs, 3 or 4 loops of the 5-loop coil were identified in the MPA after the initial coil placement. After repositioning of the coil, repeated echocardiographic examinations revealed the presence of 1 loop in the MPA. Slight residual shunting was present, but the continuous murmur had also disappeared in these 2 dogs.

\section{Discussion}

One of the challenges associated with successful placement of a transarterial coil to treat PDA in dogs is the choice of the appropriately sized coil. Estimation of the size of the coil may be inexact because of inaccuracies in the determination of the size of the shunt (ie, assessments of the smallest diameter of the shunt and the ampulla's diameter and its length); the borders of the PDA may not be clearly defined because of the close proximity of the aorta and a resultant overlap on angiograms. More reliable measurements could be performed with the use of biplane angiography. Transesophageal echocardiography is another method that could facilitate more accurate measurement of the dimensions of a ductus. ${ }^{13}$ 
Another difficulty is to define the exact position of the PDA when the tip of the delivery catheter is being positioned. Some cardiologists put marks on the fluoroscopic screen to indicate the ductus' position during display of the relevant frame of the angiogram, but in those instances, no view changes (eg, zooming in and out) or table moving may be done after the diagnostic angiographic evaluation. Another method to localize the ductus on images (after an angiogram has been obtained) is to relate its position to a combination of surrounding anatomic structures such as the trachea and certain ribs. These methods together with pressure measurements may still result in inaccurate orientation and subsequent improper coil placement. The use of digital subtraction angiography could increase the accuracy of coil positioning, but because of high costs, only a few veterinary centers have such equipment.

One of the most important radiographic signs of a properly placed coil is the presence of a gap between the loop that is in the MPA and the rest of the coil that is in the ductus. This gap is caused by the walls of the MPA and the ductus.

Pariaut et $\mathrm{al}^{13}$ investigated the use of transesophageal echocardiography in a dog with PDA. Transesophageal echocardiography provides such excellent-quality images of the PDA that one might question the simultaneous use of angiography, if a transesophageal transducer is available; unfortunately, the high cost of such a transducer allows only a few centers to use this method.

Transvenous catheterization of the ductus is an alternative approach to occlude a PDA. ${ }^{5}$ In that procedure, after an aortic angiogram, the delivery catheter is advanced through the femoral vein into the MPA and via the ductus into the aorta. The coil is pushed out of the catheter into the aorta (so that only l loop remains in the catheter), and then, together with the catheter, it is pulled back. With this method, the chance for inadvertent pulmonary artery embolization is markedly reduced because a properly sized coil cannot be pulled through the ductus. If the coil is too small and happened to be pulled through the ductus into the MPA, it could be retrieved before its detachment; the small coil can then be replaced with a larger one. Once the appropriately sized coil has lodged in the ductus, the last loop of the coil can be placed in the MPA, and the coil may be released.

Although we were not able to view the ductus itself via transthoracic echocardiography during the intervention in any of the 3 dogs of this report, the MPA and the coil could clearly be seen in each dog. Moreover, color flow Doppler imaging was able to identify residual flow through the occluded ductus in all dogs. The B-mode images obtained are not of optimal quality because the transducer is not placed on the dependent thoracic wall (small echo window because of the air-filled lungs).
In our experience, simultaneous transthoracic echocardiography helps to ensure appropriate placement (and avoid inappropriate positioning) of the transarterial coil for treatment of PDA in dogs. Betterquality images may be obtained when echocardiography is performed through the dependent thoracic wall; however, this would require a special angiographic table with a hole in it through which the ultrasound transducer could be used.

a. Martin M, Veterinary Cardiorespiratory Centre, Kenilworth, Warwickshire, United Kingdom, and Schneider M, Medical and Forensic Veterinary Clinic, Department of Small Animal Internal Medicine, Justus-Liebig University of Giessen, Giessen, Germany: Personal communications, 2005.

b. Telebrix $350,350 \mathrm{mg}$ of iodine $/ \mathrm{mL}$ (sodium ioxitalamate [4.83 g] and meglumine ioxitalamate [32.54 g]), Laboratoire Guerbet, Villepinte, France.

c. Detachable patent ductus arteriosus coil, William Cook Europe, Bjaeverskov, Denmark.

d. ATL HDI 3000, Philips, Woerden, The Netherlands

e. Surgi-Tip intraoperative transducer cover and intraoperative polyethylene ultrasound system drape kit, CIVCO Medical Instruments, Kalona, Iowa.

\section{References}

1. Buchanan JW. Causes and prevalence of cardiovascular disease. In: Kirk RW, Bonagura JD, eds. Current veterinary therapy XI: small animal practice. Philadelphia: WB Saunders Co, 1992;647-655.

2. Buchanan JW. Patent ductus arteriosus. Semin Vet Med Surg (Small Anim) 1994;9:168-176.

3. Snaps FR, Mc Entee K, Saunders JH, et al. Treatment of patent ductus arteriosus by placement of intravascular coils in a pup. J Am Vet Med Assoc 1995;207:724-725.

4. Stokhof AA, Sreeram N, Wolvekamp WTC. Transcatheter closure of patent ductus arteriosus using occluding spring coils. J Vet Intern Med 2000;14:452-455.

5. Schneider M, Hildebrandt $N$, Schweigel $T$, et al. Transvenous embolization of small patent ductus arteriosus with single detachable coils in dogs. J Vet Intern Med 2001;15:222-228.

6. Hogan DF, Green HW, Gordon S, et al. Transarterial coil embolization of patent ductus arteriosus in small dogs with 0.025-inch vascular occlusion coils: 10 cases. J Vet Intern Med 2004;18:325-329.

7. Van Israel N, French AT, Wotton PR, et al. Hemolysis associated with patent ductus arteriosus coil embolization in a dog. J Vet Intern Med 2001;15:153-156.

8. Saunders AB, Miller MW, Gordon SG, et al. Pulmonary embolization of vascular occlusion coils in dogs with patent ductus arteriosus. J Vet Intern Med 2004;18:663-666.

9. Van Israel N, French AT. Retrieval of a patent ductus arteriosus coil following embolisation to the right subclavian artery. J Small Anim Pract 2002:43:136-138.

10. Schneider M, Schneider I, Hildebrandt N, et al. Percutaneous angiography of patent ductus arteriosus in dogs: techniques, results and implications for intravascular occlusion. J Vet Cardiol 2003;5: $21-27$.

11. Glaus TM, Martin M, Boller M, et al. Catheter closure of patent ductus arteriosus in dogs: variation in ductal size requires different techniques. J Vet Cardiol 2003;5:7-12.

12. Thomas WP, Gaber CE, Jacobs GJ, et al. Recommendations for standards in transthoracic two-dimensional echocardiography in the dog and cat. J Vet Intern Med 1993;7:247-252

13. Pariaut R, Moise NS, Kraus MS, et al. Use of transesophageal echocardiography for visualization of the patent ductus arteriosus during transcatheter coil embolization. J Vet Cardiol 2004;6:32-39. 